\title{
Noninvasive prenatal testing for assessing foetal sex chromosome aneuploidy: a retrospective study of 45,773 cases
}

\author{
Xinran Lu ${ }^{1,2}$, Chaohong Wang ${ }^{2}$, Yuxiu Sun ${ }^{2}$, Junxiang Tang ${ }^{2}$, Keting Tong ${ }^{2}$ and Jiansheng Zhu $u^{1,2^{*}}$
}

\begin{abstract}
Objective: To assess the positive predictive value (PPV) of noninvasive prenatal testing (NIPT) as a screening test for sex chromosome aneuploidy (SCA) with different maternal characteristics and prenatal decisions in positive cases.

Materials and methods: We retrospectively analysed 45,773 singleton pregnancies with different characteristics that were subjected to NIPT in the Maternity and Child Health Hospital of Anhui Province. The results were validated by karyotyping. Clinical data, diagnostic results, and data on pregnancy outcomes were collected.

Results: In total, 314 cases were SCA positive by NIPT; among those, 143 underwent invasive prenatal diagnostic testing, and 58 were true-positive. Overall, the PPVs for 45,X,47,XXX, 47,XXY and 47,XYY were 12.5\%, 51.72\%, 66.67\% and $83.33 \%$, respectively. Interestingly, when only pregnant women of advanced maternal age (AMA) were screened, the PPVs for 45,X, 47,XXX, 47,XXY and 47,XYY were $23.81 \%, 53.33 \%, 78.95 \%$, and $66.67 \%$, respectively. The frequency of SCA was significantly higher in the AMA group than in the non-AMA group. The frequencies of $47, X X X$ and $47, X X Y$ were significantly correlated with maternal age.

Conclusion: NIPT performed better in predicting sex chromosome trisomies than monosomy $X$, and patients with $45, X$ positive foetuses were more eager to terminate pregnancy than those with 47,XXX and 47,XYY. AMA may be a risk factor of having a foetus with SCA. Our findings may assist in genetic counselling of AMA pregnant women. Our pre- and posttest counselling are essential for familiarizing pregnant women with the benefits and limitations of NIPT, which may ease their anxiety and enable them to make informed choices for further diagnosis and pregnancy decisions.
\end{abstract}

Keywords: Noninvasive prenatal testing, Sex chromosome aneuploidy, Positive predictive value, Advanced maternal age, High-throughput sequencing, Prenatal screening, Prenatal diagnosis

\section{Background}

Birth defects are structural deformities or functional abnormalities caused by congenital, genetic, or environmental factors [1]. Chromosomal abnormalities can be divided into chromosomal numerical abnormalities and chromosomal structural abnormalities. Chromosomal

*Correspondence: 593130772@qq.com

${ }^{1}$ Affiliated Maternity and Child Health Hospital of Anhui Medical University, Hefei, China

Full list of author information is available at the end of the article numerical abnormalities are usually a result of damage to the mitotic mechanism of cells, while structural abnormalities are a product of chromosome breaks and relocations. The phenotype of chromosomal abnormalities usually includes male infertility, spontaneous abortion, stillbirth, neonatal death, and congenital malformations. Thus, prenatal testing has become critical in preventing congenital disabilities [2].

Sex chromosome aneuploidy (SCA) refers to conditions caused by numerical abnormalities in $\mathrm{X}$ and $\mathrm{Y}$ chromosomes, such as Turner syndrome $(45, \mathrm{X})$, triple $\mathrm{X}$

c) The Author(s) 2021. This article is licensed under a Creative Commons Attribution 4.0 International License, which permits use, sharing, adaptation, distribution and reproduction in any medium or format, as long as you give appropriate credit to the original author(s) and the source, provide a link to the Creative Commons licence, and indicate if changes were made. The images or other third party material in this article are included in the article's Creative Commons licence, unless indicated otherwise in a credit line to the material. If material is not included in the article's Creative Commons licence and your intended use is not permitted by statutory regulation or exceeds the permitted use, you will need to obtain permission directly from the copyright holder. To view a copy of this licence, visit http://creativecommons.org/licenses/by/4.0/. The Creative Commons Public Domain Dedication waiver (http://creativecommons.org/publicdomain/zero/1.0/) applies to the data made available in this article, unless otherwise stated in a credit line to the data. 
syndrome (47,XXX), Klinefelter syndrome (47,XXY), and Jacob's syndrome $(47, \mathrm{XYY})$ [1]. $45, \mathrm{X}$ is a common chromosomal disorder affecting approximately 1 in 2500 to 1 in 2000 of live-born female infants [3], the common clinical manifestations of which include congenital cardiac anomalies, renal anomalies, and acquired metabolic syndrome [3, 4]. 47,XXX occurs in 1 in 1000 female births [5]. In contrast to other trisomies, most of the girls born with triple $\mathrm{X}$ chromosomes do not have a characteristic physical appearance at birth, but their heights tend to be variable. In addition, some individuals may exhibit developmental delays (speech and motor), learning or intellectual disability, and psychiatric problems $[4,5]$. 47,XXY is the most common SCA, and it occurs in 1 of every 660 males [6]. In adults, the extra X chromosome may affect testicular development, which will result in infertility and hypogonadotropic hypogonadism [6]. Males with $47, \mathrm{XXY}$ also have a higher risk of learning disabilities, developmental delays, cardiometabolic disease, typical physical symptoms and neurodevelopmental manifestations [6, 7]. 47,XYY occurs in 1 in 1000 males [8], and these males tend to have tall stature, face social obstacles, have behavioural problems, and exhibit language impairment. The cognitive phenotype of males with 47,XYY typically includes normal to mildly diminished general intelligence [8].

The phenotypes of SCA patients include a broad range of associated symptoms that vary in severity, depending on the timing of diagnosis and type of SCA $[4,5,7-9]$. The frequency of SCA is estimated to be 1 in every 500 live births. Approximately $75-90 \%$ of cases are undiagnosed during the individual's lifetime [10]. Recently, studies reported that postnatal hormone therapy could have positive effects on behavioural phenotype if applied to SCA patients early. Moreover, screening and prenatal detection of SCA can provide an opportunity for early management, there by improving the quality of life of the affected child.

Before the introduction of noninvasive prenatal testing (NIPT), amniocentesis, chorionic villus sampling, and cord blood collection were the most common tests for assessing sex chromosome abnormalities. However, this method has been associated with an increased risk of procedure-related miscarriage $(0.5-1.0 \%)$ and maternal anxiety. In 1997, Lo et al. [11]. discovered cell-free DNA derived from the $\mathrm{Y}$ chromosome in the maternal plasma of pregnant women carrying male foetuses. NIPT analyses cell-free DNA, which is a mixture of maternal DNA and a low percentage of foetal DNA believed to originate from the trophoblasts, in a pregnant woman's blood [1, 12]. NIPT uses massively parallel sequencing for prenatal foetal aneuploidy screening. Currently, with the development of high-throughput sequencing technology, NIPT has been highly recommended in clinical practice as a screening method for foetal trisomy 21 (T21), trisomy 18 (T18), and trisomy 13 (T13) among pregnant women with a high risk of abnormal serological screening test results in the second trimester $[1,13]$. Extensive studies have demonstrated the high sensitivity and specificity of NIPT for screening T21, T18, and T13, with sensitivity values of $95.9 \%, 86.5 \%$, and $77.5 \%$, respectively, and specificity values of $99.9 \%, 99.8 \%$, and $99.9 \%$, respectively [14]. Moreover, studies have reported positive predictive values (PPVs) of $65-94 \%, 47-85 \%$, and $12-62 \%$ for T21, T18, and T13, respectively [15]. In addition, NIPT has the advantage of being noninvasive, thus avoiding the $0.5-1.0 \%$ risk of miscarriage associated with amniocentesis/chorionic villus sampling $[16,17]$.

Since the implementation of the two-child policy in China in 2014, the pregnancy rate among women aged $\geq 35$ years has gradually increased [5]. At present, it is generally believed that advanced maternal age (AMA) is an important risk factor for chromosomal abnormalities [2]. An understanding of the correlation between SCA frequency and age would provide a solid basis for the development of appropriate prenatal screening and diagnostic methods.

The aim of this study was to assess the PPV of NIPT as a screening test for SCA with different maternal characteristics and prenatal decisions.

\section{Results \\ Maternal characteristics}

A total of 45,773 maternal blood samples from singleton pregnancies were collected in the Maternity and Child Health Hospital of Anhui Province between June 1,2015 , and June 30, 2019. Gestational age at the time of amniocentesis ranged from $12^{+0}$ to $26^{+6}$, and maternal age ranged from 16 to 45 years. Before undergoing NIPT, pregnant women were subjected to screening tests, which included serological screening tests and foetal ultrasonography.

In total, there were 16,921 (36.97\%) AMA (age $\geq 35$ years) cases and 19,820 (43.30\%) cases at high or critical risk for developing SCA. B-ultrasound showed that $503(1.10 \%)$ foetuses were soft markers and that $86(0.19 \%)$ foetuses had increased nuchal translucency (NT, $\geq 3 \mathrm{~mm}$ ). Moreover, 500 (1.09\%) pregnant women missed other screening opportunities, and 7943 (17.35\%) pregnant women with no clinical indications underwent NIPT (Table 1).

\section{The clinical value of NIPT for screening for foetal SCA and pregnancy outcome}

As shown in Table 2, 143 pregnant women (45.54\%) accepted the prenatal diagnosis. All patients received 
Table 1 Maternal characteristics of pregnant women who underwent NIPT

\begin{tabular}{lr}
\hline Maternal age (years) & \multicolumn{1}{c}{ Cases (\%) } \\
\hline$<30$ & $17,449(38.12 \%)$ \\
$30 \leq$ year $\leq 34$ & $11,403(24.91 \%)$ \\
$35 \leq$ year $\leq 39$ & $14,353(31.36 \%)$ \\
$>39$ & $2568(5.61 \%)$ \\
Gestational age at NIPT (weeks) & \\
$12-15$ & $4853(10.60 \%)$ \\
$16-19$ & $33,066(72.24 \%)$ \\
$20-23$ & $6740(14.72 \%)$ \\
$24-26$ & $1037(2.27 \%)$ \\
$>26$ & $77(0.17 \%)$ \\
Clinical features & \\
Advanced maternal age (age $\geq 35$ years) & $16,921(36.97 \%)$ \\
High or critical risk of serological screening & $19,820(43.30 \%)$ \\
Foetal soft markers by B-ultrasound & $503(1.10 \%)$ \\
Increased NT & $86(0.19 \%)$ \\
Missed other screening opportunities & $500(1.09 \%)$ \\
No clinical indications & $7943(17.35 \%)$ \\
\hline
\end{tabular}

the results within 3 weeks. After prenatal diagnosis, 58 pregnant women were true-positive. One hundred forty-seven pregnancies were positive for $45, \mathrm{X}$. Of them, 56 pregnancies underwent invasive prenatal testing (38.10\%). Seven cases were true-positive, with a $12.50 \%$ PPV for NIPT. Six patients with SCA terminated their pregnancies with a termination rate of $85.71 \%$. Two of them due to foetuses structurally abnormal at ultrasound. Among patients with 47,XXX, karyotype information was available for 29 out of 61 NIPT patients with results (47.54\%). Among them, 15 cases were true-positive, with a PPV of $51.72 \%$. The NIPT and karyotype analysis were fully concordant for 15 patients, of whom 3 decided to terminate their pregnancies (20\%). Among patients with 47,XXY, karyotype information was available for 39 out of 71 NIPT results with a prenatal diagnosis (54.93\%). Twentysix cases were true-positive, with a PPV of $66.67 \%$. Nineteen pregnant women decided to terminate their pregnancies (73.08\%). Karyotype information for patients with $47, \mathrm{XYY}$ was available for 12 out of 35 NIPT patients with results with a prenatal diagnosis (34.29\%). Ten cases were confirmed to be true-positive, with a PPV of $83.33 \%$. Of these ten patients, one decided to terminate their pregnancies $(10 \%)$.

Moreover, the proportions of sex chromosome trisomy and monosomy were highly different. Among 167 cases with positive screening results for sex chromosome trisomy, 51 out of 80 NIPT results were confirmed to be true-positive by invasive prenatal testing (63.75\%). Among 56 out of 147 cases of sex chromosome monosomy with a prenatal diagnosis, 7 cases were consistent with the NIPT results (12.50\%). Chi square tests were used to further examine the associations between sex chromosome trisomy and monosomy $X$. Statistical significance was observed between the two groups $\left(X^{2}=35.374, P<0.05\right)$.

After prenatal diagnosis, all patients received prenatal genetic counselling. Among the 85 patients with false-positive results, 67 were successfully followed up. They all successfully delivered their babies. A total of 171 pregnant women refused prenatal diagnosis, and 126 had effective follow-up results. Moreover, 86 pregnant women refused prenatal diagnosis due to abortion-related anxiety, and 33 pregnant women refused prenatal diagnosis due to $\mathrm{B}$-ultrasound results showing a severe foetal condition. Another 7 pregnant women experienced spontaneous abortion or stillbirth during gestation.

The PPV of SCA detected by NIPT was $40.56 \%$ (58/143), and the PPV of SCA in pregnant women in the AMA group ( $\geq 35$ years) was $50.79 \%(32 / 63)$. The PPVs were $30.43 \%(14 / 46)$ in the high or critical risk group, $40 \%(10 / 25)$ in the no clinical indications group, $25 \%(1 / 4)$ in the missed other screening opportunities group, and $33.33 \%(1 / 3)$ in the increased NT group.

A total of 85 false-positive NIPT cases were identified. Importantly, 32 cases were suspicious for an abnormal maternal ChrX karyotype, detected by NIPT, including 23 cases with an abnormal ChrX gain and 9 cases with an abnormal ChrX loss. Based on genetic

Table 2 The results of prenatal diagnosis and clinical outcome

\begin{tabular}{|c|c|c|c|c|c|c|}
\hline \multirow[t]{2}{*}{ SCA type } & \multirow[t]{2}{*}{ NIPT positive (N) } & \multicolumn{3}{|c|}{ Karyotype analysis } & \multirow[t]{2}{*}{ Unverified (N) } & \multirow{2}{*}{$\begin{array}{l}\text { Termination } \\
\text { rate (\%) }\end{array}$} \\
\hline & & True positive (N) & False positive (N) & PPV (\%) & & \\
\hline $45, x$ & 147 & 7 & 49 & 12.5 & 91 & 85.71 \\
\hline $47, X X X$ & 61 & 15 & 14 & 51.72 & 33 & 20 \\
\hline $47, X X Y$ & 71 & 26 & 13 & 66.67 & 32 & 73.08 \\
\hline $47, X Y Y$ & 35 & 10 & 2 & 83.33 & 22 & 10 \\
\hline
\end{tabular}


counselling, 32 cases underwent maternal peripheral blood karyotyping. Moreover, 2 cases among 23 cases with an abnormal ChrX gain were diagnosed with 47,XXX, while 2 cases among 9 cases with an abnormal ChrX loss were diagnosed with mosaicism 45,X/47,XXX and mosaicism 47,XXX/45,X/46,XY.

In our study, a pregnant woman was referred for NIPT at 16 weeks gestation because of advanced maternal age, which turned to be a positive result for $45, \mathrm{X}$. The woman decided to undertake amniocentesis at $17^{+6}$ weeks, and a normal result was identified. G-banded karyotyping analysis was also carried out on peripheral blood lymphocytes derived from the couple and ruled out abnormal chromosome karyotype. Then with normal ultrasound, the women chose to continue pregnancy. The case delivered live birth at 39 weeks, and foetal and placental tissue was retained for measurement of suspected SCA mosaicism by DNA sequencing. Placental biopsies taken at the center of the tissue and at the middle and edge of the maternal-fetal interface, had variable $45, \mathrm{X}$ mosaicism levels of $70 \%, 50 \%$, and $70 \%$, respectively. The examination of the placental tissue at three sites showed $45, \mathrm{X}$ mosaicism.

\section{Relationship between SCA frequency and maternal age}

We evaluated the relationships between different PPVs and maternal age. For SCA, the PPV increased with maternal age, and was the highest among women aged $>39$ years. In our study, there were 16,921 (36.97\%) patients with AMA ( $\geq 35$ years), accounting for a relatively large population. The PPV, which was $50.79 \%$ for

Table 3 The NIPT results of SCA between AMA and nonAMA groups

\begin{tabular}{lllll}
\hline Age & SCA & Non-SCA & OR $(95 \% \mathrm{Cl})$ & P value \\
\hline AMA & 32 & 16,840 & $2.098(1.250-3.521)$ & 0.004 \\
Non-AMA & 26 & 28,704 & & \\
\hline
\end{tabular}

SCA in AMA women, was slightly higher than that of all samples (40.56\%). The frequency of SCA (32/16872) in the AMA group was significantly higher than in the non-AMA group $(26 / 28730) \quad\left(\chi^{2}=8.229, \quad P=0.004\right.$, $\mathrm{OR}=2.098,95 \%$ CI- 1.250 to 3.521 ) (Table 3 ). The frequencies of $45, \mathrm{X}$ and $47, \mathrm{XYY}$ did not differ among age groups of pregnant women $\left(X^{2}=4.328, P>0.05\right.$ for $45, X$, and $X^{2}=1.074, P>0.05$ for $\left.47, X Y Y\right)$. However, the frequencies of $47, \mathrm{XXX}$ and $47, \mathrm{XXY}$ were different among the different age groups of $\left(X^{2}=12.616, P<0.05\right.$ for $47, \mathrm{XXX}$, and $\mathrm{X}^{2}=12.570, P<0.05$ for $47, \mathrm{XXY}$ ) (Table 4 ).

\section{Discussion}

Several recent studies reported the sensitivity, specificity, and PPV of NIPT for T21, T18, and T13 screening. However, to date, no large-scale clinical studies have been conducted to assess the efficiency of NIPT for detecting SCA. To the best of our knowledge, this is the largest study (45,773 cases) to evaluate NIPT for SCA. According to our data, the overall PPV of SCA detected by NIPT was $40.56 \%$, within the range of PPV for SCA screening between 30 and $60 \%$ [12, 13, 15, 18, 19]. When categorized by individual SCAs, the PPVs were $12.5 \%$ for $45, \mathrm{X}$, $51.72 \%$ for $47, \mathrm{XXX}, 66.67 \%$ for $47, \mathrm{XXY}$, and $83.33 \%$ for $47, \mathrm{XYY}$, which were similar to those reported by other studies $[1,13,20]$. However, in our study, we noticed that different pregnancy characteristics showed different PPVs; for example, the PPV of SCA in AMA pregnancy cases $(50.79 \%)$ was higher than that in all cases (40.56\%). Moreover, the overall termination rate was $50 \%$ for SCA (when mosaic cases were included) and $41.38 \%$ (when mosaic cases were not included); the termination rates for foetal SCA were $85.71 \%$ for $45, \mathrm{X}, 20 \%$ for $47, \mathrm{XXX}$, $73.08 \%$ for $47, \mathrm{XXY}$, and $10 \%$ for $47, \mathrm{XYY}$ [13].

Our study showed that NIPT performed better in predicting sex chromosome trisomies than monosomy $\mathrm{X}$. This may be due to the following. [1] There are 1098 genes on the $\mathrm{X}$ chromosome and 78 genes on the $\mathrm{Y}$ chromosome, and 58 genes are homologous genes on

Table 4 NIPT results for SCA screening in pregnant women of different age groups

\begin{tabular}{|c|c|c|c|c|c|c|c|c|c|c|c|c|c|c|c|c|c|c|}
\hline \multirow[t]{2}{*}{ NIPT results } & \multicolumn{3}{|c|}{ All cases } & \multicolumn{3}{|c|}{ AMA cases } & \multicolumn{3}{|c|}{ Age $<30$ years } & \multicolumn{3}{|c|}{$30 \leq$ age $\leq 34$ years } & \multicolumn{3}{|c|}{$35 \leq$ age $\leq 39$ years } & \multicolumn{3}{|c|}{ Age $>39$ years } \\
\hline & TP & FP & PPV & TP & FP & PPV & TP & $\mathrm{FP}$ & PPV & $\mathrm{TP}$ & FP & PPV & TP & FP & PPV & $\mathrm{TP}$ & FP & PPV \\
\hline $45, x$ & 7 & 49 & 12.5 & 5 & 16 & 23.81 & 2 & 18 & 10 & 0 & 15 & 0 & 4 & 14 & 22.22 & 1 & 2 & 33.33 \\
\hline $47, X X X$ & 15 & 14 & 51.72 & 8 & 7 & 53.33 & 4 & 4 & 50 & 3 & 3 & 50 & 4 & 5 & 44.44 & 4 & 2 & 66.67 \\
\hline $47, X X Y$ & 26 & 13 & 66.67 & 15 & 4 & 78.95 & 4 & 6 & 40 & 7 & 3 & 70 & 10 & 4 & 71.43 & 5 & 0 & 100 \\
\hline $47, X Y Y$ & 10 & 2 & 83.33 & 4 & 2 & 66.67 & 3 & 0 & 100 & 3 & 0 & 100 & 4 & 2 & 66.67 & / & / & / \\
\hline SCA suspected abnormal & 0 & 7 & & 0 & 2 & & 0 & 2 & & 0 & 3 & & 0 & 2 & & / & / & / \\
\hline Total & 58 & 85 & 40.56 & 32 & 31 & 50.79 & 13 & 30 & 30.23 & 13 & 24 & 35.14 & 22 & 27 & 44.9 & 10 & 4 & 71.43 \\
\hline
\end{tabular}


both sex chromosomes. The majority of the genes (29 genes) are at the ends of the sex chromosomes. [2] The low guanosine-cytosine content of the $\mathrm{X}$ chromosome leads to highly variable amplification of the $\mathrm{X}$ chromosome. [3] The nonrandom inactivation of the $\mathrm{X}$ chromosome in placental tissue might be the reason for the low PPV of Turner syndrome, with the paternal X chromosome tending to be inactivated in XX female trophoblasts $[1,13]$. In addition, it was reported that there is an age-related $\mathrm{X}$ chromosome loss in normal female white blood cells, which may influence the effectiveness of predicting foetal 45,X [13], although this was not observed in our study.

The PPV of SCA is lower than that of other common chromosome aneuploidies. The reason is that sex chromosome abnormalities are less prevalent [15]. Wang et al. reported that $8.6 \%$ of positive results for SCA were due to maternal mosaicism [21], which was later confirmed by other studies $[1,13,22]$. With the combination of NIPT and maternal peripheral blood karyotype analysis, we found that approximately $12.5 \%$ of the discordant NIPT SCA results were due to maternal mosaicism in our study. Previous studies have demonstrated that the identification of maternal karyotypes tends to decrease the rate of false-positive SCA and can offer an explanation for the false-positive results for SCA $[13,14,23]$. With regard to the discordance between NIPT and invasive prenatal testing, another reason is confined placental mosaicism (CPM), which occurs in approximately $1-2 \%$ of all pregnancies [12]. The origin of most cell-free foetal DNA (cffDNA) in the maternal plasma is mostly the apoptosis of placental cells from the cytotrophoblast [24]. The mosaicism degree reduces the effective cffDNA concentration in maternal plasma, thus affecting the performance of NIPT in detecting foetal aneuploidies. Grati et al. determined the potential contribution of CPM to the NIPT false-positive rate, demonstrating that chromosomes 13 and $\mathrm{X}$ were more likely to be associated with CPM than chromosomes 18 and 21 [25]. In current study, postnatal placental analysis was preformed in one case with CPM. This case illustrates that extensive cytogenetic analysis can be required to identify $C P M$, and that patients should be counseled regarding the possibility of discordant NIPT results. However, obtaining the placenta for clinical cases remains challenge for clinical laboratories preforming analysis of discordant NIPT results. Our results verified the value of determining the maternal karyotype and examination of placental tissue in increasing the accuracy of reporting NIPT results for chromosomes $\mathrm{X}$ and $\mathrm{Y}$; however, further studies are needed to provide more clinical data in support of this premise.
Despite the prenatal diagnosis of foetal SCA, some pregnant women were willing to continue their pregnancies, and there were differences in the rates of pregnancy termination between different types of SCAs. Pregnant women whose foetuses were $45, \mathrm{X}$ or $47, \mathrm{XXY}$ were more eager to terminate their pregnancies than those whose foetuses were $47, \mathrm{XXX}$ or $47, \mathrm{XYY}$, which was consistent with other studies [13, 26]. Gruchy et al. [27] reported that with regard to Turner syndrome, the rate of pregnancy termination was closely related to the $45, \mathrm{X}$ karyotype, mosaic karyotype, and structural abnormalities of the $\mathrm{X}$ chromosome. In our study, pregnant women whose foetuses were $45, \mathrm{X}$ with mosaicism of $23.08 \%$ had a stronger tendency to continue their pregnancies, while those with mosaicism of $28.38 \%, 48.53 \%, 59.38 \%, 76.67 \%$, and $80.49 \%$ were more inclined to terminate their pregnancies. Current studies report that almost all pregnant women carrying foetuses with $45, \mathrm{X}$ decide to terminate their pregnancy [28]. To some extent, the parental decisions for pregnancy termination may have been related to the types of SCA, level of prenatal genetic counselling, history of infertility, parental and social acceptance, economic condition, and related factors.

In clinical practice, AMA pregnant women are willing to accept NIPT as a noninvasive and accurate screening method. In this study, the proportion of AMA pregnant women was the second highest in the NIPT screening population, reaching $36.97 \%$. Among them, NIPT detected 108 cases of SCA; 63 cases underwent amniocentesis, and $32(50.79 \%)$ cases were confirmed to be true-positive. Of all the confirmed SCA cases, 55.17\% $(32 / 58)$ were AMA pregnant women. The study showed that NIPT could be used as a useful screening test for SCA in AMA pregnant women, which was similar to the results reported by Zheng et al. [29]. The differences in the frequency of SCA were statistically significant among the age groups, and the frequency was significantly higher in the $>39$ years age group $(P<0.05)$. And the frequency of SCA in the AMA group was significantly higher than in the non-AMA group. We may conclude that AMA may be a risk factor for SCA. Therefore, genetic counselling, combined with serological screening tests and B-ultrasound detection of abnormalities, should be fully carried out for AMA pregnant women. The frequencies of 47,XXX and 47,XXY were significantly correlated with maternal age, whereas the frequencies of $45, \mathrm{X}$ and $47, \mathrm{XYY}$ did not show significant correlations. Although there was no statistical significance regarding the frequency of 47,XYY and maternal age, the frequency of $47, \mathrm{XYY}$ decreased with maternal age in the advanced aged group. To determine whether there is a correlation between maternal age and the frequency of a foetus 
with 47,XYY, further studies with larger sample sizes are warranted [2]. The risk of 47,XXX increased with AMA, which was consistent with the findings by Zhu et al. [2]. Previous studies showed that for $45, \mathrm{X}$ syndrome, the maternal age coefficient is negative, implying a decreasing frequency among older mothers [2]. However, this was not consistent with our results. Understanding the frequency of SCA has proven valuable in counselling couples who seek advice about the risk of foetal sex chromosome abnormalities with AMA and are considering the option of prenatal diagnosis and termination of pregnancy.

This study has a few limitations. First, the sensitivity, specificity, and negative predictive value were not calculated. New-borns with SCA usually appear phenotypically normal. Therefore, it was difficult to confirm the results of SCA without karyotype analysis during the neonatal period. Second, the number of SCA cases in our study was not large enough to discuss its frequency across different age groups. Therefore, more pregnancies must be evaluated to further understand the association between maternal age and foetal SCA.

Recent studies have demonstrated that early interventions such as postnatal hormone therapy, physical therapy, and occupational therapy could have positive effects on the behavioural phenotype or neurodevelopmental outcomes if applied earlier to SCA patients $[9,30]$. Prenatal screening and diagnosis of SCA can provide the opportunity for early intervention, comprehensive postnatal management, and improve the quality of life of the affected child [10]. Sex chromosome abnormalities are more common than major trisomies at birth, and neonates are often phenotypically normal [31]. Conventional prenatal screening cannot be used to directly identify sex chromosome abnormalities that can only be identified using postnatal karyotyping, which in turn may delay the treatment of SCA patients. NIPT allows prenatal screening of SCA. The application of NIPT can allow pregnant women to have an alternate option to invasive prenatal testing for the identification of foetal sex chromosome abnormalities. However, there are still some issues that require further consideration. Due to the existing false-positive rate of NIPT screening for SCA, the number of unnecessary invasive prenatal diagnoses may increase, especially for 45,X [13]. Nevertheless, the benefit of detection for foetal SCA outweighs the risk related to invasive procedures. Some pregnant women may decide to terminate their pregnancies if chromosomal abnormalities are incidentally discovered by SCA screening, which involves ethical issues regarding the mild phenotype of the SCA and the potential increase in the rate of sex selection [13].
With the development of sequencing technology, recent methods have higher accuracy than earlier methods which used to rely on chromosome dosage changes only. Huang et al. [32] presented a novel silicon-based nanostructured microfluidic platform (Cell Reveal ${ }^{\mathrm{TM}}$ ) for capturing circulating fetal nucleated red blood cells (fnRBC) and extravillous cytotrophoblasts (EVT) for cell-based noninvasive prenatal diagnosis (cbNIPD). This method used a microfluidic device coated with specific antibodies to capture the related antigen covered cells. Through the method, the foetal cells could be isolated from the maternal circulation and foetal DNA could be used efficiently to detect copy number abnormalities [32]. In addition, another method, named cell-based noninvasive prenatal testing, could be used to detect the subchromosomal abnormalities $(\geq 1 \mathrm{Mb})$ in foetal cells by using low-coverage shotgun next-generation sequencing [33], which was an ideal way to balance the depth and accuracy of sequencing. Moreover, whole-genome NGS methylomic analysis was also a method to provide a version of the placental methylome from the maternal plasma that could be effective for chromosomal abnormalities testing [34]. All together, NIPT's research on chromosomal abnormalities has been expanding and deepening, not only staying on the chromosome dosage changes.

\section{Conclusions}

Our data suggest that NIPT can be used to identify foetal SCA by analysing cffDNA obtained from maternal plasma using massively parallel sequencing technology. We found that patients with $45, \mathrm{X}$ foetuses were more eager to terminate their pregnancies. Different maternal characteristics have different PPVs. AMA may be a risk factor for SCA. Additionally, the frequencies of 47,XXX and 47,XXY were correlated with maternal age. Pre- and posttest counselling are essential to familiarizing women with the benefits and limitations of NIPT, which may ease their anxiety and enable them to make an informed choice for further diagnosis and pregnancy decisions.

\section{Materials and methods \\ Subjects}

In this study, 45,773 women with singleton pregnancies who underwent NIPT at the Maternity and Child Health Hospital of Anhui Province between June 1, 2015, and June 30, 2019, were recruited. Maternal age, serological screening results, NT, and results were recorded. The inclusion criteria were as follows: [1] gestational week between $12^{+0} \sim 26^{+6}$ and [2] singleton pregnancy. The exclusion criteria were as follows: [1] gestational age $<12$ weeks; [2] multiple pregnancies; [3] definite chromosomal abnormalities; [4] pregnant women who 
underwent an allogeneic blood transfusion, stem cell therapy, transplant surgery, or other procedure; [5] a family history of genetic disease or an indication for a high risk of genetic disease in the foetus; [6] pregnant women with malignant tumours; and [7] other conditions that might affect the accuracy of the results.

The study was approved by the Ethics Committee of Anhui Medical University. Informed consent was obtained from all patients. Before testing, all patients received a consultation with a genetic counsellor or clinical geneticist, after which a prenatal diagnosis was recommended.

\section{Maternal serum screening tests and ultrasonography}

Maternal age $\geq 35$ years was defined as AMA [2]. We used a combination of the first trimester screening (from 9 weeks to $13^{+6}$ weeks) and the second trimester screening (from 15 weeks to $20^{+6}$ weeks). The serological screening tests used were pregnancy-associated plasma protein A (PAPP-A) and free $\beta$-HCG for the first trimester screening and AFP, free $\beta$-HCG, and free E3 for the second trimester screening. A time-resolved immunofluorescence assay was used for detection. The risk values were calculated by Lifecycle software (4.0): high risk, T21 >1/300, T18>1/350; critical risk, $\mathrm{T} 21=$ from $1 / 300$ to $1 / 1000, \mathrm{~T} 18=$ from $1 / 350$ to $1 / 1000$. The following signs were mainly used as B-ultrasound soft markers: absent or shortened nasal bone, thickened nuchal fold, single umbilical artery, echogenic bowel, pyelectasis, and choroid plexus cysts [35, 36]. NT was measured by a trained sonographer following the Foetal Medicine Foundation protocol, and NT $\geq 3 \mathrm{~mm}$ was defined as increased NT [37].

\section{Noninvasive prenatal testing (NIPT)}

A total of $10 \mathrm{~mL}$ of maternal peripheral blood was collected in a Cell-Free DNA BCTTM tube (EDTA). Plasma was separated from the maternal plasma by two rounds of centrifugation within $48 \mathrm{~h}$. Whole blood was centrifuged at $1600 \times \mathrm{g}$ for $10 \mathrm{~min}$ at $4{ }^{\circ} \mathrm{C}$, after which the supernatant was centrifuged at $16000 \times \mathrm{g}$ for $10 \mathrm{~min}$. The maternal plasma was immediately stored at $-80{ }^{\circ} \mathrm{C}$ until DNA extraction. Extraction of cffDNA, library construction, quality control, and pooling were performed in the laboratory of Maternity and Child Health Hospital of Anhui Province. DNA was extracted by the QIAamp Circulating Nucleic Acid Kit (Qiagen). The pooled library was sequenced using an Illumina with the Data Analysis System for bioinformatic analysis of the sequencing data. The sequencing reads were filtered and aligned to the human reference genome (hg19) [15]. Calculating a Z score per chromosome and chromosome with an absolute value of $\mathrm{Z}$ score $>3$ was identified with chromosome aneuploidies or microdeletions/microduplications [38].
Additionally, samples with a normalized chromosome value of 3.0 or less were marked as normal.

\section{Karyotype analysis of amniotic fluid}

Karyotype analysis of amniotic fluid was performed for samples collected from pregnant women who were positive for SCA by NIPT and who agreed to undergo invasive prenatal diagnosis, which is the gold standard for chromosome aneuploidy testing. Briefly, under B-ultrasound guidance, $20 \mathrm{~mL}$ of amniotic fluid was aseptically withdrawn using amniocentesis. Inoculation, culture, G-banding, and karyotype scanning using a GSL-120 automatic karyotype scanner were then performed. The karyotype was described according to the "International System for Human Cytogenetic Nomenclature, ISCN2016" guidelines. According to the principle outlined by the American College of Medical Genetics and Genomics, 2018, a total of 30 dividing phases were counted per sample using an AI chromosome image analysis system (CytoVision, Switzerland). Five karyotypes were analysed, and double counts were obtained in the case of chimaeras.

\section{Statistical analysis}

Statistical analysis was performed using SPSS version 23 (IBM Corp., Armonk, NY, USA). A Chi square test was applied to compare the frequencies of SCA among pregnant women in different age groups. A $P$ value of $<0.05$ was considered to be statistically significant.

\section{Supplementary information}

The online version contains supplementary material available https://doi. org/10.1186/s13039-020-00521-2.

Additional file 1: Supplementary materials

\section{Acknowledgements}

We are very thankful to the patients who had provided their peripheral blood cells and amniotic fluid for this study. We are also grateful to the technical support of doctors and paramedic staff of Maternity and Child Health Hospital, Hefei, Anhui, PR China.

\section{Author's contribution}

LX designed the study and drafted the manuscript. WC and SY collected all the data. TJ analysed and interpreted the data. TK participated in the laboratory workflow. ZJ oversaw the work and revised the manuscript. All authors have read and approved the final manuscript.

\section{Funding}

Not applicable.

\section{Availability of data and materials}

The datasets used and/or analysed during the current study are available from the corresponding author on reasonable request.

\section{Competing interests}

The authors have declared no conflicts of interest.

Ethics approval and consent to participate

The study was approved by the Ethics Committee of Anhui Medical University. 


\section{Author details}

${ }^{1}$ Affiliated Maternity and Child Health Hospital of Anhui Medical University, Hefei, China. ${ }^{2}$ Maternity and Child Health Hospital of Anhui Province, Hefei, China.

Received: 15 September 2020 Accepted: 9 December 2020

Published online: 06 January 2021

\section{References}

1. Zhang B, Lu BY, Yu B, et al. Noninvasive prenatal screening for fetal common sex chromosome aneuploidies from maternal blood. J Int Med Res. 2017:45(2):621-30.

2. Zhu Y, Lu S, Bian X, et al. A multicenter study of fetal chromosomal abnormalities in Chinese women of advanced maternal age. Taiwan J Obstet Gynecol. 2016;55(3):379-84.

3. Mavridi A, Ntali G, Theodora M, Stamatelopoulos K, Michala L. A spontaneous pregnancy in a patient with turner syndrome with $45, X / 47, X X X$ Mosaicism: a case report and review of the literature. J Pediatr Adolesc Gynecol. 2018;31(6):651-4.

4. Lim HH, Kil HR, Koo SH. Incidence, puberty, and fertility in $45, X / 47, X X X$ mosaicism: report of a patient and a literature review. Am J Med Genet A. 2017;173(7):1961-4.

5. Rafique M, AlObaid S, Al-Jaroudi D. 47, XXX syndrome with infertility, premature ovarian insufficiency, and streak ovaries. Clin Case Rep. 2019;7(6):1238-41.

6. Davis SM, Rogol AD, Ross JL. Testis development and fertility potential in boys with klinefelter syndrome. Endocrinol Metab Clin North Am. 2015;44(4):843-65.

7. Samango-Sprouse CA, Counts DR, Tran SL, Lasutschinkow PC, Porter GF, Gropman AL. Update on the clinical perspectives and care of the child with 47, XXY (Klinefelter Syndrome). Appl Clin Genet. 2019;12:191-202.

8. Matsuzaki J, Bloy L, Blaskey L, et al. Abnormal auditory mismatch fields in children and adolescents with 47. XYY Syndrom Dev Neurosci. 2019;41(1-2):123-31.

9. Samango-Sprouse C, Lasutschinkow P, Powell S, et al. The incidence of anxiety symptoms in boys with 47, XXY (Klinefelter syndrome) and the possible impact of timing of diagnosis and hormonal replacement therapy. Am J Med Genet A. 2019;179(3):423-8.

10. Samango-Sprouse CA, Porter GF, Lasutschinkow PC, et al. Impact of early diagnosis and noninvasive prenatal testing (NIPT): knowledge, attitudes, and experiences of parents of children with sex chromosome aneuploidies (SCAs). Prenat Diagn. 2020;40(4):470-80

11. Lo YM, Corbetta N, Chamberlain PF, Rai V, Sargent IL, Redman CW, Wainscoat JS. Presence of fetal DNA in maternal plasma and serum. Lancet. 1997;350(9076):485-7.

12. Chen Y, Yu Q, Mao X, Lei W, He M, Lu W. Noninvasive prenatal testing for chromosome aneuploidies and subchromosomal microdeletions/ microduplications in a cohort of 42,910 single pregnancies with different clinical features. Hum Genom. 2019;13(1):60.

13. $X u Y$, , Chen $L$, Liu $Y$, Hao $Y, X u Z$, Deng $L$, Xie J. Screening, prenatal diagnosis, and prenatal decision for sex chromosome aneuploidy. Exp Rev Molecular Diagnost. 2019;19(6):537-42.

14. Taylor-Phillips S, Freeman K, Geppert J, et al. Accuracy of non-invasive prenatal testing using cell-free DNA for detection of Down, Edwards and Patau syndromes: a systematic review and meta-analysis. BMJ Open. 2016;6(1):e010002.

15. Hu H, Wang L, Wu J, et al. Non-invasive prenatal testing for chromosome aneuploidies and sub chromosomal microdeletions/microduplications in a cohort of 8141 single pregnancies[J]. Human Genom. 2019;13(1):1-9.

16. Cuckle H, Benn P, Pergament EJCB. Cell-free DNA screening for fetal aneuploidy as a clinical service. Clin Biochem. 2015;48(15):932-41.

17. Bevilacqua E, Ordóñez E, Hurtado I, et al. Screening for sex chromosome aneuploidy by cell-free DNA testing: patient choice and performance. Fetal Diagn Ther. 2018;44(2):98-104.

18. Petersen AK, Cheung SW, Smith $J$, et al. Positive predictive value estimates for cell-free noninvasive prenatal screening from data of a large referral genetic diagnostic laboratory. Am J Obstet Gynecol. 2017;217(6):691.e1-6.
19. Wang Y, Li S, Wang W, Dong Y, Zhang M, Wang X, Yin C. Cell-free DNA screening for sex chromosome aneuploidies by non-invasive prenatal testing in maternal plasma. Mol Cytogenet. 2020;12(13):10.

20. Ramdaney A, Hoskovec J, Harkenrider J, et al. Clinical experience with sex chromosome aneuploidies detected by noninvasive prenatal testing (NIPT): accuracy and patient decision making. Prenat Diag. 2018;38:841-8.

21. Wang Y, Chen Y, Tian F, et al. Maternal mosaicism is a significant contributor to discordant sex chromosomal aneuploidies associated with noninvasive prenatal testing. Clin Chem. 2014;60(1):251-9.

22. Song Y, Liu C, Qi H, et al. Noninvasive prenatal testing of fetal aneuploidies by massively parallel sequencing in a prospective Chinese population. Prenat Diag. 2013;38:700-6.

23. Lau TK, Jiang FM, Stevenson RJ, et al. Secondray finding from non-invasive prenatal testing for common fetal aneuploidies by whole genome sequencing as a clinical service. Print Diagn. 2013;33:602-8.

24. Taglauer ES, Wilkins-Haug L, Bianchi DW. Review: cell-free fetal DNA in the maternal circulation as an indication of placental health and disease. Placenta. 2014;35:S64-8.

25. Grati FR, Bajaj K, Malvestiti F, et al. The type of feto-placental aneuploidy detected by cfDNA testing may influence the choice of confirmatory diagnostic procedure. Prenat Diagn. 2015;35(10):994-8.

26. So PL, Cheng KYY, Cheuk KY, et al. Parental decisions following prenatal diagnosis of sex chromosome aneuploidy in Hong Kong. J Obstet Gynaecol Res. 2017;43(12):1821-9.

27. Gruchy N, Vialard F, Blondeel E, et al. Pregnancy outcomes of prenatally diagnosed turner syndrome: a French multicenter retrospective study including a series of 975 cases. Prenat Diagn. 2014;34(12):1133-8.

28. Zhou Q, Zhu ZP, Zhang B, Yu B, Cai ZM, Yuan P. Clinical features and pregnancy outcomes of women with abnormal cell-free fetal DNA test results. Ann Transl Med. 2019;7(14):317.

29. Zheng J, Lu H, Li M, et al. The clinical utility of non-invasive prenatal testing for pregnant women with different diagnostic indications. Front Genet. 2020;11:624.

30. Howard-Bath A, Poulton A, Halliday J, et al. Population-based trends in the prenatal diagnosis of sex chromosome aneuploidy before and after non-invasive prenatal testing. Prenat Diagn. 2018;38(13):1062-8.

31. Nicolaides KH, Musci TJ, Struble CA, Singelaki A, Gil MM. Assessment of fetal sex chromosome aneuploidy using directed cell-free DNA analysis. Fetal Diagn Ther. 2014;35:1-6.

32. Huang CE, Ma GC, Jou HJ, et al. Noninvasive prenatal diagnosis of fetal aneuploidy by circulating fetal nucleated red blood cells and extravillous trophoblasts using silicon-based nanostructured microfluidics. Mol Cytogenet. 2017;10:44.

33. Vossaert L, Wang Q, Salman R, et al. Reliable detection of subchromosomal deletions and duplications using cell-based noninvasive prenatal testing. Prenat Diagn. 2018;38(13):1069-78.

34. Sun K, Lun FMF, Leung TY, et al. Noninvasive reconstruction of placental methylome from maternal plasma DNA: potential for prenatal testing and monitoring. Prenat Diagn. 2018;38(3):196-203.

35. Ahman A, Axelsson O, Maras G, Rubertsson C, Sarkadi A, Lindgren P. Ultrasonographic fetal soft markers in a low-risk population: prevalence, association with trisomies and invasive tests. Acta Obstet Gynecol Scand. 2014;93(4):367-73.

36. Hsiao $\mathrm{CH}$, Cheng PJ, Shaw SW, et al. Extended first-trimester screening using multiple sonographic markers and maternal serum biochemistry: a five-year prospective study. Fetal Diagn Ther. 2014;35(4):296-301.

37. Maya I, Yacobson S, Kahana S, et al. Cut-off value of nuchal translucency as indication for chromosomal microarray analysis. Ultrasound Obstet Gynecol. 2017;50(3):332-5.

38. Zhang H, Zhao YY, Song J, et al. Statistical approach to decreasing the error rate of noninvasive prenatal aneuploid detection caused by maternal copy number variation. Sci Rep. 2015;5:16106.

\section{Publisher's Note}

Springer Nature remains neutral with regard to jurisdictional claims in published maps and institutional affiliations. 